\title{
46,XX/46,XY Ovotesticular Differences of Sex Development
}

National Cancer Institute

\section{Source}

National Cancer Institute. 46, XX/46,XY Ovotesticular Differences of Sex Development. NCI Thesaurus. Code C127174.

Ovotesticular differences of sex development in individuals with 46,XY/46,XX mosaic karyotype. 